\title{
232.
}

\section{MÉMOIRE SUR LA FORME CANONIQUE DES FONCTIONS BINAIRES.}

[From the Journal für die reine und angewandte Mathematik, (Crelle), tom. LIV. (1857), pp. $48-58$.

L'IDÉE de la forme canonique d'une fonction binaire de degré impair et la théorie de la réduction d'une pareille fonction à la forme canonique sont dues à M. Sylvester $\left(^{1}\right)$, qui a, en outre, étendu sa théorie aux fonctions binaires des degrés pairs 4 et 6 . Mais il n'a établi nulle part l'idée générale de la forme canonique d'une fonction biuaire d'un degré pair quelconque. Je me propose de reprendre cette théorie en considérant d'abord les fonctions binaires de degré impair et ensuite les fonctions binaires de degré pair.

Soit donc proposée la fonction de degré impair

$$
\left(a, b, \ldots a^{\prime} \gamma x, y\right)^{2 m-1}
$$

en désignant par le symbole $\Sigma$ une somme de $m$ termes, on voit qu'on pourra écrire

$$
\left(a, b, \ldots a^{\prime} \gamma x, y\right)^{2 m-1}=\Sigma A(x+\alpha y)^{2 m-1},
$$

l'expression qui forme le second membre de cette équation est appelée la forme canonique de la fonction donnée, et il s'agit de trouver les valeurs des quantités $A, \alpha$.

En supposant que le produit des facteurs linéaires $x+\alpha y$ soit égal, à un facteur constant près, à

$$
\left(\mathrm{a}, \mathrm{b}, \ldots \mathrm{a}^{\prime} \gamma x, y\right)^{m}
$$

${ }^{1}$ Les recherches de M. Sylvester sont contenues dans le mémoire intitulé "Sketch of a Memoir on Elimination, Transformation, and Canonical Forms," Camb. and Dublin Mathematical Journal, t. vi. [1851] p. 186, dans un supplément publié conjointement avec ce mémoire et intitulé "Essay on Canonical Forms by J. J. Sylvester," London, Bell, 1851, et dans le mémoire intitulé, "On a remarkable discovery in the Theory of Canonical Forms and of Hyperdeterminants," Phil. Mag. Nov. 1851. 
la question peut s'énoncer sous la forme suivante: trouver une fonction de degré $m$, telle qu'en représentant $x+a y$ un facteur linéaire quelconque de cette fonction, la fonction donnée de degré $2 m-1$ se réduise à la forme $\Sigma A(x+\alpha y)^{2 m-1}$. La fonction de degré $m$ contiendra un facteur constant dont la valeur peut être prise à volonté. Mais à ce facteur près la fonction de degré $m$ sera complètement déterminée.

Pour fixer les idées je suppose que la fonction donnée soit la fonction cubique

$$
\left(a, b, c, d \gamma(x, y)^{3},\right.
$$

dans ce cas il s'agira de trouver une fonction quadratique

$$
\left(\mathrm{a}, \mathrm{b}, \mathrm{c} \gamma(x, y)^{2}\right.
$$

telle qu'en posant $\left(\mathrm{a}, \mathrm{b}, \mathrm{c} \gamma(x, y)^{2}=\mathrm{a}(x+\alpha y)(x+\beta y)\right.$ on ait identiquement

$$
\left(a, b, c, d \gamma(x, y)^{3}=A(x+\alpha y)^{3}+B(x+\beta y)^{3} .\right.
$$

Cela se fait très-facilement par les méthodes ordinaires. En effet, on a

d'où il suit

$$
\begin{aligned}
& A+B=a \\
& A \alpha+B \beta=b \\
& A \alpha^{2}+B \beta^{2}=c \\
& A \alpha^{3}+B \beta^{3}=d
\end{aligned}
$$

$$
\begin{aligned}
& a \xi \eta-b(\xi+\eta)+c=A(\xi-\alpha)(\eta-\alpha)+B(\xi-\beta)(\eta-\beta), \\
& b \xi \eta-c(\xi+\eta)+d=A \alpha(\xi-\alpha)(\eta-\alpha)+B \beta(\xi-\beta)(\eta-\beta),
\end{aligned}
$$

$\xi$ et $\eta$ étant des quantités quelconques; donc, en prenant $\xi=\alpha, \eta=\beta$, on obtient

$$
\begin{aligned}
& a \alpha \beta-b(\alpha+\beta)+c=0, \\
& b \alpha \beta-c(\alpha+\beta)+d=0 .
\end{aligned}
$$

Mais l'équation $\left(\mathrm{a}, \mathrm{b}, \mathrm{c} \gamma(x, y)^{2}=\mathrm{a}(x+\alpha y)(x+\beta y)\right.$ donne

$$
\alpha \beta: \alpha+\beta: 1=\mathrm{c}: 2 \mathrm{~b}: \mathrm{a},
$$

on a donc

$$
\begin{aligned}
& a c-2 b b+c a=0, \\
& b c-2 c b+d a=0,
\end{aligned}
$$

système qui donne les rapports $\mathrm{a}: \mathrm{b}: \mathrm{c}$. Mais pour compléter la solution de la manière la plus élégante, il faut ajouter à ces équations, l'équation $\left(a, b, c \gamma(x, y)^{2}=0\right.$. mise sous la forme $(\mathrm{c}, \mathrm{b}, \mathrm{a} \gamma y, x)^{2}=0$, ou ce qui est la même chose

$$
y^{2} \mathrm{c}+2 y x \mathrm{~b}+x^{2} \mathrm{a}=0 ;
$$


en éliminant $\mathrm{a}, \mathrm{b}, \mathrm{c}$ on obtient une équation de laquelle on déduit

$$
\left(\mathrm{a}, \mathrm{b}, \mathrm{c} \gamma(x, y)^{2}=\left|\begin{array}{ccc}
y^{2}, & -y x, & x^{2} \\
a, & b & c \\
b, & c, & d
\end{array}\right| .\right.
$$

Mais on peut trouver ce résultat d'une manière encore plus facile. Considérant l'équation

$$
\left(a, b, c, d \gamma(x, y)^{3}=A(x+a y)^{3}+B(x+\beta y)^{3},\right.
$$

on n'a qu'à opérer sur cette équation en se servant du symbole

$$
\left(\mathrm{a}, \mathrm{b}, \mathrm{c} \gamma \partial_{y},-\partial_{x}\right)^{2}
$$

le second membre se réduit à zéro à cause des équations $\left(\mathrm{a}, \mathrm{b}, \mathrm{c} \gamma(\alpha,-1)^{2}=0,(\mathrm{a}, \mathrm{b}, \mathrm{c} \gamma \beta,-1)^{2}=0\right.$ que donne l'équation $\left(\mathrm{a}, \mathrm{b}, \mathrm{c} \gamma(x, y)^{2}=\mathrm{a}(x+\alpha y \gamma(x+\beta y)\right.$. Par conséquent on a identiquement

c'est-à-dire

$$
\left(\mathrm{a}, \mathrm{b}, \mathrm{c} \gamma\left(\partial_{y},-\partial_{x}\right)^{2}\left(a, b, c, d \gamma(x, y)^{3}=0,\right.\right.
$$

ou enfin

$$
x(a c-2 b b+c a)+y(b c-2 c b+d a)=0,
$$

$$
\begin{aligned}
& a c-2 b b+c a=0, \\
& b c-2 c b+d a=0,
\end{aligned}
$$

et de là on tire comme auparavant la valeur de la fonction $\left(\mathrm{a}, \mathrm{b}, \mathrm{c} \gamma(x, y)^{2}\right.$.

Les valeurs des coefficients $A, B$ s'expriment alors en fonctions linéaires des coefficients de la fonction cubique, et des quantités $\alpha, \beta$ qui sont pour ainsi dire les racines de la fonction quadratique $\left(\mathrm{a}, \mathrm{b}, \mathrm{c} \gamma(x, y)^{2}\right.$.

Il est évident que le procédé suivi dans ce qui précède est tout à fait général, et que l'on obtient toujours explicitement la fonction du degré $m$ : par exemple pour la fonction du cinquième degré

la fonction dont il s'agit sera

$$
\left(a, b, c, d, e, f \gamma(x, y)^{5}\right.
$$

$$
\left|\begin{array}{rrrr}
y^{3}, & -y^{2} x, & y x^{2}, & -x^{3} \\
a, & b, & c, & d \\
b, & c, & d, & e \\
c, & d, & e, & f
\end{array}\right|
$$

et de même dans tous les autres cas. Cette fonction du degré $m$ (laquelle est un covariant de la fonction donnée) peut être appelée le canonisant. Pour la fonction du cinquième degré le canonisant peut être mis sous cette autre forme

$$
\left|\begin{array}{lll}
a x+b y, & b x+c y, & c x+d y \\
b x+c y, & c x+d y, & d x+e y \\
c x+d y, & d x+e y, & e x+f y
\end{array}\right|
$$

et on démontre facilement qu'il existe toujours une transformation semblable. 
Considérons maintenant une fonction de degré pair

$$
\left(a, b, \ldots a^{\prime} \gamma(x, y)^{2 m}\right.
$$

et posons

$$
\left(a, b, \ldots a^{\prime} \gamma(x, y)^{2 m}=\Sigma A(x+\alpha y)^{2 m}+\Lambda\left(a, b, \ldots a^{\prime} \chi x, y\right)^{m}\left(a_{1}, b_{1}, \ldots a_{1}^{\prime} \gamma(x, y)^{m}\right.\right.
$$

où l'on suppose $\left(a, b, \ldots a^{\prime} \gamma(x, y)^{m}=a(x+\alpha y) \ldots\right.$ et où $\left(a_{1}, b_{1}, \ldots a_{1}^{\prime} \gamma(x, y)^{m}\right.$ représente un certain covariant parfaitement déterminé de $\left(a, b, \ldots a^{\prime} \chi x, y\right)^{m}$. L'expression qui forme le second membre de l'équation proposée s'appellera la forme canonique de la fonction de degré pair. degré

Pour fixer les idées je suppose que la fonction donnée soit la fonction du quatrième

$$
\left(a, b, c, d, e \gamma(x, y)^{4}\right.
$$

dans ce cas, en prenant la fonction quadratique $\left(\mathrm{a}, \mathrm{b}, \mathrm{c} \gamma(x, y)^{2}=\mathrm{a}(x+\alpha y)(x+\beta y)\right.$ et en représentant par $\left(\mathrm{a}_{1}, \mathrm{~b}_{1}, \mathrm{c}_{1} \chi(x, y)^{2}\right.$ un covariant déterminé de $\left(\mathrm{a}, \mathrm{b}, \mathrm{c} \gamma(x, y)^{2}\right.$ on a

$$
\left(a, b, c, d, e \gamma(x, y)^{4}=A(x+\alpha y)^{4}+B(x+\beta y)^{4}+\Lambda\left(a, b, c \gamma(x, y)^{2}\left(a_{1}, b_{1}, c_{1}\right)(x, y)^{2} .\right.\right.
$$

Cela posé, la condition qui sert à déterminer le covariant $\left(a_{1}, b_{1}, c_{1}\right)(x, y)^{2}$ est la suivante

$$
\frac{1}{2}\left(\mathrm{a}, \mathrm{b}, \mathrm{c} \gamma \partial_{y},-\partial_{x}\right)^{2} \cdot\left(\mathrm{a}, \mathrm{b}, \mathrm{c} \gamma(x, y)^{2}\left(\mathrm{a}_{1}, \mathrm{~b}_{1}, \mathrm{c}_{1} \chi(x, y)^{2}=K\left(\mathrm{a}, \mathrm{b}, \mathrm{c} \gamma(x, y)^{2},\right.\right.\right.
$$

où $K$ est un facteur constant dont la valeur est arbitraire.

Le premier membre sera

$$
\left(\begin{array}{ccc}
7 a c-6 b^{2}, & -2 a b, & a^{2}, \\
2 b c, & 12 a c-16 b^{2}, & 2 a b, \\
c^{2}, & -2 b c, & \left.\left.7 a c-6 b^{2}, b_{1}, c_{1}\right)\right)(x, y)^{2}
\end{array} \mid\right.
$$

en représentant par cette notation l'expression

$$
\begin{aligned}
& {\left[\left(7 \mathrm{ac}-6 \mathrm{~b}^{2}\right) \mathrm{a}_{1} \quad-2 \mathrm{abb}_{1} \quad+\mathrm{a}^{2} \mathrm{c}_{1}\right] x^{2}} \\
& +\left[2 b^{2} a_{1}+\left(12 a c-16 b^{2}\right) b_{1}+2 a b c_{1}\right] x y \\
& +\left[\begin{array}{lll}
\mathrm{c}^{2} \mathrm{a}_{1} & -2 \mathrm{bcb}_{1}+\left(7 \mathrm{ac}-6 \mathrm{~b}^{2}\right) \mathrm{c}_{1}
\end{array}\right] y^{2} \text {. }
\end{aligned}
$$

On a donc

$$
\begin{aligned}
& \left(7 \mathrm{ac}-6 \mathrm{~b}^{2}\right) \mathrm{a}_{1} \quad-2 \mathrm{abb}_{1} \quad+\mathrm{a}^{2} \mathrm{c}_{1}=K \mathrm{a} \text {, } \\
& 2 \mathrm{bca}_{1}+\left(12 \mathrm{ac}-16 \mathrm{~b}^{2}\right) \mathrm{b}_{1}+2 \mathrm{abc}_{1}=K 2 \mathrm{~b}, \\
& c^{2} a_{1}-2 b c b_{1}+\left(7 a c-6 b^{2}\right) c_{1}=K c,
\end{aligned}
$$

et en ajoutant à ces équations la suivante

$$
x^{2} \mathrm{a}_{1}+2 x y \mathrm{~b}_{1}+y^{2} \quad \mathrm{c}_{1}=u,
$$


on obtient, en éliminant $a_{1}, b_{1}, c_{1}$, l'équation

$\left|\begin{array}{cccc}u, & x^{2}, & 2 x y \quad, & y^{2} \\ K a, & 7 a c-6 b^{2}, & -2 a b \quad, & a^{2} \\ 2 K b, & 2 b c & 12 a c-16 b^{2}, & 2 a b \\ K c, & c^{2}, & -2 b c \quad, & 7 a c-6 b^{2}\end{array}\right|=0$

laquelle peut s'écrire aussi comme il suit

$$
\begin{array}{ccc}
7 \mathrm{ac}-6 \mathrm{~b}^{2}, & -2 \mathrm{ab}, & \mathrm{a}^{2} \\
2 \mathrm{bc}, & 12 \mathrm{ac}-16 \mathrm{~b}^{2}, & 2 \mathrm{ab} \\
\mathrm{c}^{2}, & -2 \mathrm{bc}, & 7 \mathrm{ac}-6 \mathrm{~b}^{2}
\end{array}|+| \begin{array}{rrrr}
x^{2} & 2 x y, & y^{2} \\
\mathrm{a}, & 7 \mathrm{ac}-6 \mathrm{~b}^{2}, & -2 \mathrm{ab}, & \mathrm{a}^{2} \\
2 \mathrm{~b}, & 2 \mathrm{bc}, & 12 \mathrm{ac}-16 \mathrm{~b}^{2}, & 2 \mathrm{ab} \\
\mathrm{c}, & \mathrm{c}^{2}, & -2 \mathrm{bc}, & 7 \mathrm{ac}-6 \mathrm{~b}^{2}
\end{array} \mid=0
$$

donc, en rétablissant la valeur de $u$, savoir $u=\left(\mathrm{a}_{1}, \mathrm{~b}_{1}, \mathrm{c}_{1} \gamma x, y\right)^{2}$, et en prenant

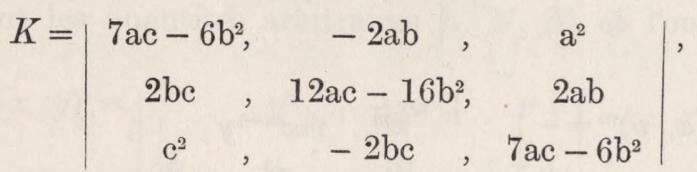

on obtient

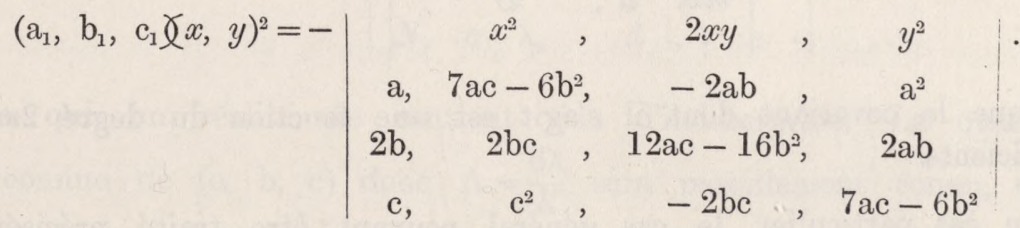

Ces valeur's se réduisent à des expressions très simples. En effet, on a $K=576\left(\mathrm{ac}-\mathrm{b}^{2}\right)^{3}$, $\left(a_{1}, b_{1}, c_{1} \gamma(x, y)^{2}=-72\left(a c-b^{2}\right)^{2}(a, b, c \gamma x, y)^{2}\right.$, de sorte qu'en supprimant le facteur constant $-72\left(a c-b^{2}\right)^{2}$ on peut prendre

$$
\left(\mathrm{a}_{1}, \mathrm{~b}_{1}, \mathrm{c}_{1} \gamma x, y\right)^{2}=\left(\mathrm{a}, \mathrm{b}, \mathrm{c} \gamma(x, y)^{2} .\right.
$$

C'est ce que l'on aurait pu prévoir dès le commencement; car le procédé par lequel on obtient $\left(a_{1}, b_{1}, c_{1} \gamma x, y\right)^{2}$ fait voir que cette fonction est un covariant de $(a, b, c \gamma x, y)^{2}$, du second degré par rapport aux variables et du cinquième degré par rapport aux coefficients; donc cette fonction sera, à un facteur numérique près, identique avec $\left(\mathrm{ac}-\mathrm{b}^{2}\right)^{2}\left(\mathrm{a}, \mathrm{b}, \mathrm{c} \gamma(x, y)^{2}\right.$. Une circonstance pareille se présente dans la réduction d'une fonction du sixième degré à la forme canonique. En effet le covariant $\left(a_{1}, b_{1}, c_{1}, d_{1} \gamma x, y\right)^{2}$ sera une fonction du troisième degré par rapport aux variables et du septième degré par rapport aux coefficients, donc à un facteur numérique près, cette fonction sera identique avec le discriminant $\left(\mathrm{a}^{2} \mathrm{~d}^{2}+\right.$ etc.) multiplié par le cubicovariant $\left(\mathrm{a}^{2} \mathrm{~d}-3 \mathrm{abc}+2 \mathrm{~b}^{3}\right) x^{3}+$ etc., ou, en supprimant le facteur constant, le covariant cherché sera tout simplement le cubicovariant de $\left(\mathrm{a}, \mathrm{b}, \mathrm{c}, \mathrm{d} \gamma(x, y)^{3}\right.$. De même pour la fonction du huitième degré on 
trouve que le covariant $\left(\mathrm{a}_{1}, \mathrm{~b}_{1}, \mathrm{c}_{1}, \mathrm{~d}_{1}, \mathrm{e}_{1} \chi x, y\right)^{4}$ sera tout simplement $\left(\mathrm{a}, \mathrm{b}, \mathrm{c}, \mathrm{d}, \mathrm{e} \gamma(x, y)^{4}\right.$. Mais rien ne nous autorise à supposer qu'une réduction de cette espèce ait lieu dans le cas général, et il paraît qu'en général le covariant $\left(a_{1}, b_{1}, \ldots a_{1}^{\prime} \backslash x, y\right)^{m}$ sera une fonction indécomposable du degré $2 m+1$ par rapport aux coefficients; à savoir, en posant

$$
\left(a, b, \ldots a^{\prime} \gamma \partial_{y},-\partial_{x}\right)^{m}\left(a, b, \ldots a^{\prime}(x, y)^{m}\left(a_{1}, b_{1} \ldots a_{1}^{\prime}(x, y)^{m}=K\left(a, b, \ldots a^{\prime} \gamma(x, y)^{m}\right.\right.\right.
$$

le premier membre sera une fonction de la forme

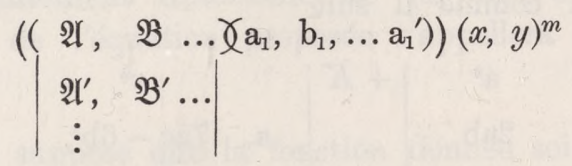

où $\mathfrak{A}, \mathfrak{B}, \ldots \mathfrak{A}^{\prime}, \mathfrak{B}^{\prime}, \ldots$ etc. sont des fonctions données du second degré par rapport aux coefficients $\left(a, b, \ldots a^{\prime}\right)$; cela étant, on aura

$$
K=\left|\begin{array}{cc}
\mathfrak{A}, & \mathfrak{B}, \ldots \\
\mathfrak{A}^{\prime}, & \mathfrak{B}^{\prime}, \ldots \\
\vdots &
\end{array}\right|
$$

et

$$
\left(\mathrm{a}_{1}, \mathrm{~b}_{1}, \ldots \mathrm{a}_{1}^{\prime}(x, y)^{m}=-\left|\begin{array}{rccc} 
& x^{m}, & m x^{m-1} y \\
\mathrm{a}, & \mathfrak{A}, & \mathfrak{B} & \ldots \\
m \mathrm{~b}, & \mathfrak{A}^{\prime}, & \mathfrak{B}^{\prime} & \\
\vdots & &
\end{array}\right|,\right.
$$

ce qui fait voir que le covariant dont il s'agit est une fonction du degré $2 m+1$ par rapport aux coefficients.

Je reviens au cas particulier, le cas général pouvant être traité précisément de la même manière. On a

$$
(a, b, c, d, e \chi x, y)^{4}=A(x+\alpha y)^{4}+B(x+\beta y)^{4}+\Lambda\left(a, b, c \gamma(x, y)^{2}\left(\mathrm{a}_{1}, \mathrm{~b}_{1}, \mathrm{c}_{1} \chi(x, y)^{2} .\right.\right.
$$

J'opère sur cette équation en me servant du symbole $\left(a, b, c \gamma \partial_{y},-\partial_{x}\right)^{2}$ et j'obtiens

$$
\left(\mathrm{a}, \mathrm{b}, \mathrm{c} \gamma \partial_{y},-\partial_{x}\right)^{2}\left(a, b, c, d, e_{\chi} x, y\right)^{4}=2 \Lambda K\left(\mathrm{a}, \mathrm{b}, \mathrm{c} \gamma(x, y)^{2}\right. \text {, }
$$

c'est-à-dire

$$
\left.\begin{array}{r}
a\left(c x^{2}+2 d x y+e y^{2}\right) \\
-2 b\left(b x^{2}+2 c x y+d y^{2}\right) \\
+\quad c\left(a x^{2}+2 b x y+c y^{2}\right)
\end{array}\right\}=\frac{1}{6} \Lambda K\left(a x^{2}+2 b x y+c y^{2}\right)
$$

en posant, pour abréger, $\frac{1}{6} \Lambda K=\lambda$, on aura

$$
\begin{aligned}
& \left.\begin{array}{lll}
x^{2}[\mathrm{c} a & -2 \mathrm{~b} b & +\mathrm{a}(c-\lambda)
\end{array}\right] \\
& +2 x y\left[\begin{array}{lll}
\mathrm{c} b & -2 \mathrm{~b}\left(c+\frac{1}{2} \lambda\right)+\mathrm{a} d \quad
\end{array}\right]=0 \text {; } \\
& +y^{2}[c(c-\lambda)-2 b d \quad+a e \quad]
\end{aligned}
$$


ce qui donne

$$
\begin{array}{ll}
\mathrm{c} a+2 \mathrm{~b} b+\mathrm{a}(c-\lambda)=0 \\
\mathrm{c} b-2 \mathrm{~b}\left(c+\frac{1}{2} \lambda\right)+\mathrm{a} d=0 \\
\mathrm{c}(c-\lambda)-2 \mathrm{~b} d+\mathrm{a} e & =0
\end{array}
$$

et de là on tire l'équation

$$
\left|\begin{array}{ccc}
a, & b, & c-\lambda \\
b, & c+\frac{1}{2} \lambda, & d \\
c-\lambda, & d, & e
\end{array}\right|=0
$$

qui servira à la détermination de la quantité $\lambda$. Les coefficients des différentes puissances de $\lambda$ seront des invariants; en effet, si on développe le déterminant, l'équation devient $2\left(a c e+2 b c d-a d^{2}-b^{2} e+c^{3}\right)+\lambda\left(a e-4 b d+3 c^{2}\right)-\lambda^{3}=0$. La quantité $\lambda$ étant connue, on peut au moyen de deux quelconques des trois équations trouver les rapports a : b : c, ou ce qui revient à la même chose, on peut se servir des trois équations en introduisant les quantités arbitraires $L, M, N$, et l'on trouve

$$
\left(\mathrm{a}, \mathrm{b}, \mathrm{c}(x, y)^{2}=\left|\begin{array}{cccc} 
& y^{2}, & -y x, & x^{2} \\
L, & a, & b, & c-\lambda \\
M, & b, & c+\frac{1}{2} \lambda, & d \\
N, & c-\lambda, & d, & e
\end{array}\right|\right.
$$

expression qui, en réalité, ne contient rien d’indéterminé. La quantité $K$ est une fonction connue de (a, b, c) donc $\Lambda=\frac{6 \lambda}{K}$ sera pareillement connu, et les coefficients $A, B$ s'expriment en fonctions linéaires des coefficients de la fonction du quatrième degré et des quantités $\alpha, \beta$ qui sont pour ainsi dire les racines de la fonction quadratique $\left(\mathrm{a}, \mathrm{b}, \mathrm{c} \gamma(x, y)^{2}\right.$. Comme je l'ai déjà fait observer, tout ce qui précède s'applique également à une fonction de degré pair quelconque. Le déterminant qui contient $\lambda$ peut être appelé le Lambdaïque. On voit aisément quelle est sa forme générale; par exemple pour la fonction $\left(a, b, c, d, e, f, g \gamma(x, y)^{6}\right.$ ce déterminant est

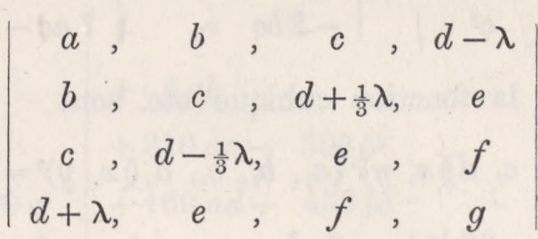

expression dont le développement ne contient que les puissances paires de $\lambda$; cela arrive, comme M. Sylvester l'a remarqué depuis longtemps, toutes les fois que $m$ est impair, c'est-à-dire toutes les fois que le degré de la fonction dont il s'agit est un nombre de la forme $4 p+2$.

C. IV. 
Revenons à la fonction du quatrième degré $\left(a, b, c, d, e \gamma(x, y)^{4}\right.$; en écrivant $\left(\mathrm{a}_{1}, \mathrm{~b}_{1}, \mathrm{c}_{1} \gamma(x, y)^{2}=\left(\mathrm{a}, \mathrm{b}, \mathrm{c} \gamma(x, y)^{2}=\mathrm{a}(x+\alpha y)(x+\beta y)\right.\right.$ on obtient pour la forme canonique

$$
\left(a, b, c, d, e \gamma(x, y)^{4}=A(x+\alpha y)^{4}+B(x+\beta y)^{4}+\Lambda(x+\alpha y)^{2}(x+\beta y)^{2} .\right.
$$

Relativement à la fonction du sixième degré $\left(a, b, c, d, e, f, g \gamma(x, y)^{6}\right.$, je fais observer que le cubicovariant de $(x+\alpha y)(x+\beta y)(x+\gamma y)$ est

$$
\begin{array}{r}
\{(2 \alpha-\beta-\gamma) x-(2 \beta \gamma-\gamma \alpha-\alpha \beta) y\}\{(2 \beta-\gamma-\alpha) x-(2 \gamma \alpha-\alpha \beta-\beta \gamma) y\} \\
\{(2 \gamma-\alpha-\beta) x-(2 \alpha \beta-\beta \gamma-\gamma \alpha) y\} ;
\end{array}
$$

donc en représentant cette fonction par $\Phi$, on a pour la forme canonique

$$
\begin{aligned}
\left(a, b, c, d, e, f, g \gamma(x, y)^{6}\right. & \\
& =A(x+\alpha y)^{6}+B(x+\beta y)^{6}+C(x+\gamma y)^{6}+\Lambda(x+\alpha y)(x+\beta y)(x+\gamma y) \Phi .
\end{aligned}
$$

Pour la fonction du huitième degré le covariant $\left(a_{1}, b_{1}, c_{1}, d_{1}, e_{1} \chi x, y\right)^{4}$ est tout simplement égal à $(\mathrm{a}, \mathrm{b}, \mathrm{c}, \mathrm{d}, \mathrm{e}\}(x, y)^{4}$ ainsi que je l'ai déjà fait observer, et l'on a pour la forme canonique

$$
\left(a, b, c, d, e, f, g, h, i \gamma(x, y)^{8}\right.
$$

$=A(x+\alpha y)^{8}+B(x+\beta y)^{8}+C(x+\gamma y)^{8}+D(x+\delta y)^{8}+\Lambda(x+\alpha y)^{2}(x+\beta y)^{2}(x+\gamma y)^{2}(x+\delta y)^{2}$.

Je n'ai pas cherché à réduire, ou à présenter sous la forme d'un déterminant, le covariant $\left(a_{1}, b_{1}, c_{1}, d_{1}, e_{1}, f_{1} \chi_{x}, y\right)^{5}$ qui entre dans la forme canonique de la fonction du dixième degré.

On a vu que le covariant $\left(a_{1}, b_{1}, \ldots a_{1}^{\prime} \chi x, y\right)^{m}$ s'obtient par le développement de la fonction

$$
\left(\mathrm{a}, \mathrm{b}, \ldots \mathrm{a}^{\prime} \gamma \partial_{y},-\partial_{x}\right)^{m} \cdot\left(\mathrm{a}, \mathrm{b}, \ldots \mathrm{a}_{1}^{\prime} \chi(x, y)^{m}\left(\mathrm{a}_{1}, \mathrm{~b}_{1}, \ldots \mathrm{a}_{1}^{\prime} \gamma(x, y)^{m}\right.\right.
$$

et j'ai donné ci-dessus un exemple de ce développement; en employant pour plus de commodité les lettres italiques au lieu des lettres romaines, on peut écrire la formule dont il s'agit de la manière suivante

$$
\begin{aligned}
& \left(a, b, c \gamma \partial_{y},-\partial_{x}\right)^{2}\left(a, b, c \gamma(x, y)^{2}\left(a_{1}, b_{1}, c_{1} \chi x, y\right)^{2}=\right. \\
& 2\left(\begin{array}{l|l|l}
7 a c-6 b^{2} & -2 a b \\
2 b c & 12 a c-16 b^{2} & \left|\begin{array}{l}
a^{2} \\
2 a b \\
7 a c-6 b^{2}
\end{array}\right| \\
c^{2} & -2 b c & \left.\left(a_{1}, b_{1}, c_{1}\right)\right)(x, y)^{2} .
\end{array}\right.
\end{aligned}
$$

Les équations analogues pour la fonction cubique etc. sont

$$
\begin{aligned}
& \left(a, b, c, d \gamma \partial_{y},-\partial_{x}\right)^{3}\left(a, b, c, d \gamma(x, y)^{3}\left(a_{1}, b_{1}, c_{1}, d_{1} \gamma(x, y)^{3}=\right.\right. \\
& 6 \times\left(\left(\begin{array}{l|l|l|l}
-19 a d+18 b c & +39 a c-36 b^{2} & -3 a b & +a^{2} \\
-39 b d+36 c^{2} & -18 a d+27 b c & +72 a c-81 b^{2} & +3 a b \\
-3 c d & -72 b d+81 c^{2} & +18 a d-27 b c & +39 a c-36 b^{2} \\
-d^{2} & +3 c d & -39 b d+36 c^{2} & +19 a d-18 b c
\end{array} \mid\right.\right.
\end{aligned}
$$


$\left(a, b, c, d, e \gamma \partial_{y},-\partial_{x}\right)^{4}\left(a, b, c, d, e \gamma(x, y)^{4}\left(a_{1}, b_{1}, c_{1}, d_{1}, e_{1} \gamma x, y\right)^{4}=\right.$

$$
\begin{aligned}
& 24 \times\left(\left(+71 a e-160 b d+90 c^{2}|-124 a d+120 b c \quad|+126 a c-120 b^{2}\right.\right. \\
& +124 b e-120 c d \quad+160 a e-896 b d+720 c^{2}-120 a d+144 b c \\
& +126 c e-120 d^{2} \quad+120 b e-144 c d \quad+180 a e-1440 b d+1296 c^{2} \\
& \begin{array}{l|l|l}
+4 d e & +240 c e-256 d^{2} & -120 b e+144 c d
\end{array} \\
& 1+e^{2} \quad 1+4 d e \quad 126 c e-120 d^{2} \\
& \begin{array}{l|ll}
-4 a b & +a^{2} \\
+240 a c-256 b^{2} & +4 a b \\
+120 a d-144 b c & \left.\left(a_{1}, b_{1}, c_{1}, d_{1}, e_{1}\right)\right)(x, y)^{4} . \\
+160 a e-896 b d+720 c & +126 a c-120 b^{2} \\
-124 b e+120 c d & +71 a e-160 b d+90 c^{2}
\end{array} \mid
\end{aligned}
$$$$
\left(a, b, c, d, e, f \gamma \partial_{y},-\partial_{x}\right)^{5}\left(a, b, c, d, e, f \gamma(x, y)^{5}\left(a_{1}, b_{1}, c_{1}, d_{1}, e_{1}, f_{1} \gamma x, y\right)^{5}=\right.
$$

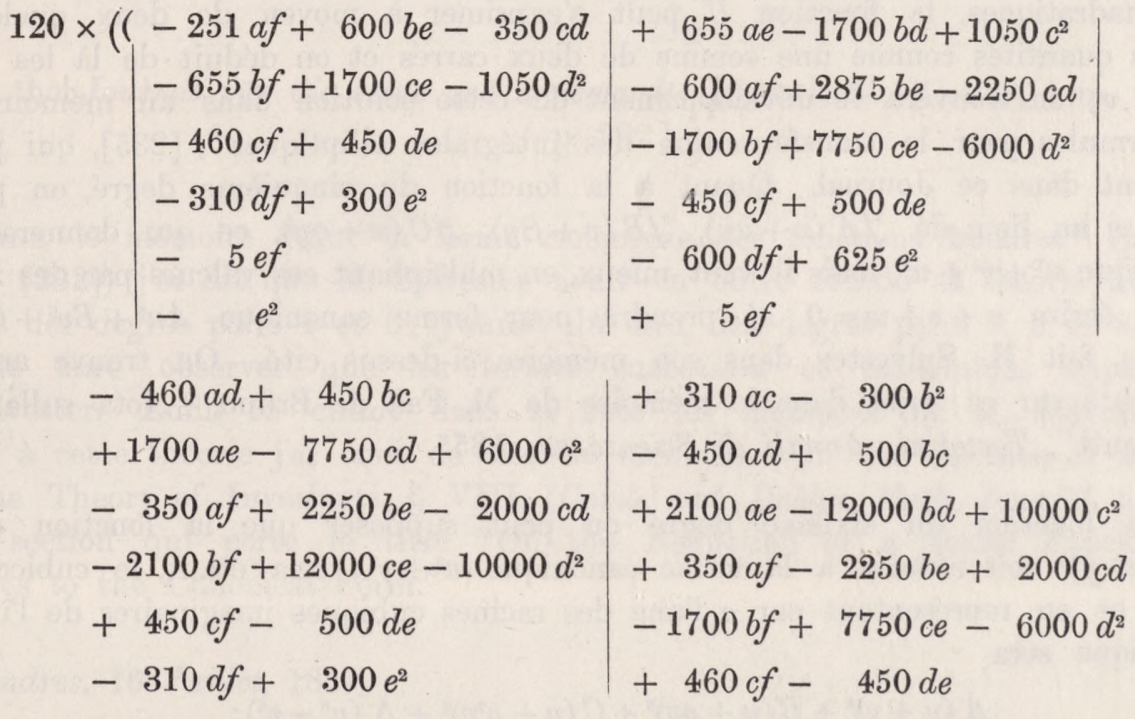$$
\begin{array}{l|l}
-5 a b & +a^{2} \\
+600 a c-625 b^{2} & +5 a b \\
+450 a d-500 b c & +310 a c-300 b^{2} \\
+1700 a e-7750 b d+6000 c^{2} & +460 a d-450 b c \\
+600 a f-2875 b e+2250 c d & +655 a e-1700 b d+1050 c^{2} \\
-655 b f+1700 c e-1050 d^{2} & +251 a f-600 b e+350 c d
\end{array} \mid
$$

Les résultats qu'on vient d'obtenir pour les cas particuliers les plus simples font voir que la théorie générale doit être susceptible de développements ultérieurs. Ainsi la 
forme canonique de la fonction cubique $\left(a, b, c, d \gamma(x, y)^{3}\right.$ sera $u^{3}+v^{3}$ si l'on pose $u, v$ au lieu de $\sqrt[3]{A}(x+\alpha y), \sqrt[3]{B(x+\beta y)}$. La méthode générale ne donne que la valeur du produit $u, v$ à un facteur constant près; mais on peut trouver les valeurs de $u$ et $v$ séparément. En effet en se rappelant les résultats obtenus dans la "Note sur les covariants d'une fonction quadratique, cubique, ou biquadratique à deux indéterminées" (t. L. p. 285 de ce Journal, [135]), on reconnaît que les deux fonctions $\Phi+U \sqrt{ }-\square, \Phi-U \sqrt{ }-\square$ sont l'une et l'autre des cubes parfaits et l'on a

$$
2 U \sqrt{ }-\square=(\Phi+U \sqrt{ }-\square)-(\Phi-U \sqrt{ }-\square)
$$

on a donc

$$
u=\left\{\frac{\Phi+U \sqrt{ }-\square}{2 \sqrt{ }-\square}\right\}^{\frac{1}{3}}, \quad v=\left\{\frac{\Phi-U \sqrt{ }-\square}{2 \sqrt{ }-\square}\right\}^{\frac{1}{2}}
$$

De même la forme canonique de la fonction du quatrième degré $(a, b, c, d, e \gamma x, y)^{4}$ sera $u^{4}+v^{4}+6 \theta u^{2} v^{2},=\left(u^{2}-v^{2}\right)^{2}+2(1+3 \theta) u^{2} v^{2}$, si l'on pose $u, v$ au lieu de $\sqrt[4]{A}(x+\alpha y)$,

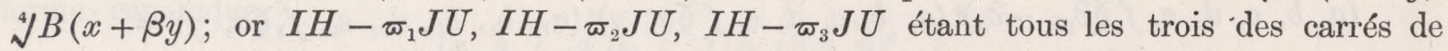
fonctions quadratiques, la fonction $U$ peut s'exprimer à moyen de deux quelconques de ces trois quantités comme une somme de deux carrés et on déduit de là les valeurs de $u$ et de $v$; on trouvera le développement de cette solution dans un mémoire "Sur quelques formules pour la transformation des intégrales elliptiques", [235], qui paraîtra prochainement dans ce Journal. Quant à la fonction du cinquième degré, on pourrait écrire $u, v, w$ au lieu de $\sqrt[5]{A}(x+\alpha y), \sqrt[5]{ } B(v+\beta y), \sqrt[5]{C}(x+\gamma y)$, ce qui donnerait pour forme canonique $u^{5}+v^{5}+w^{5}$, mais il vaut mieux, en multipliant ces valeurs par des facteurs convenables, écrire $u+v+w=0$ et prendre pour forme canonique $A u^{5}+B v^{5}+C w^{5}=0$, c'est ce qu'a fait M. Sylvester dans son mémoire ci-dessus cité. On trouve aussi des développements sur ce sujet dans le mémoire de M. Faà de Bruno, "Nota sulla teorica degli invarianti", Tortolini, Annali di Scienze etc. 1855.

Pour la fonction du sixième degré on peut supposer que la fonction cubique $\left(\mathrm{a}, \mathrm{b}, \mathrm{c}, \mathrm{d} \gamma(x, y)^{3}\right.$ soit réduite à la forme canonique $u^{3}+v^{3}$; cela étant, le cubicovariant sera $u^{3}-v^{3}$, et en représentant par $\rho$ l'une des racines cubiques imaginaires de l'unité la forme canonique sera

$$
A(u+v)^{6}+B(u+\rho v)^{6}+C\left(u+\rho^{2} v\right)^{6}+\Lambda\left(u^{6}-v^{6}\right) ;
$$

c'est encore ce qu'a fait voir M. Sylvester dans son mémoire.

Londres, 9 Avril, 1856. 